\title{
BUOYANCY OF THE "Y2K" PERSISTENT TRAIN AND THE TRAJECTORY OF THE 04:00:29 UT LEONID FIREBALL
}

\author{
PETER JENNISKENS \\ SETI Institute, NASA ARC, Mail Stop 239-4, Moffett Field, California 94035 \\ E-mail: pjenniskens@mail.arc.nasa.gov \\ and \\ RICK L. RAIRDEN \\ Lockheed Martin Space Sciences LaboratoryDept L9-42, Bldg 255, 3251 Hanover \\ Street, Palo Alto, California 94304 \\ E-mail: rairden@spasci.com \\ (Received 23 June 2000; Accepted 29 July 2000)
}

\begin{abstract}
The atmospheric trajectory is calculated of a particularly well studied fireball and train during the 1999 Leonid Multi-Instrument Aircraft Campaign. Less than a minute after the meteor's first appearance, the train curves into a "2"-shape, which persisted until at least 13 minutes after the fireball. We conclude that the shape results because of horizontal winds from gravity waves with a scale height of $8.3 \mathrm{~km}$ at $79-91$ $\mathrm{km}$ altitude, as well as a westerly wind gradient with altitude. In addition, there is downward drift that affects the formation of loops in the train early on.
\end{abstract}

Keywords: Fireball, leonids 1999, lower thermosphere, mesosphere, meteor, persistent train, winds

\section{Introduction}

A bright fireball of absolute magnitude about -13 appeared over the isle of Corsica at 04:00:29 UT in the night of November 18, 1999. The fireball registered on three slit-less spectrographs onboard the Leonid Multi-Instrument Aircraft Campaign, probing various wavelength ranges in the near-UV, visual and optical near-IR. The fireball provided the first spectrum of a meteor's afterglow, which made it possible to study the cooling rate of the emitting gas in the first seconds after the meteor (Borovicka and Jenniskens, 2000). Once the afterglow had subsided, a luminous glow persisted for more than 13 minutes. Such persistent trains have eluded a better understanding for over a century (Lockeyer, 1869). 
Especially the latter aspect is of interest in understanding the physical conditions in the meteor path. We have examined the observations for evidence of such vertical motions

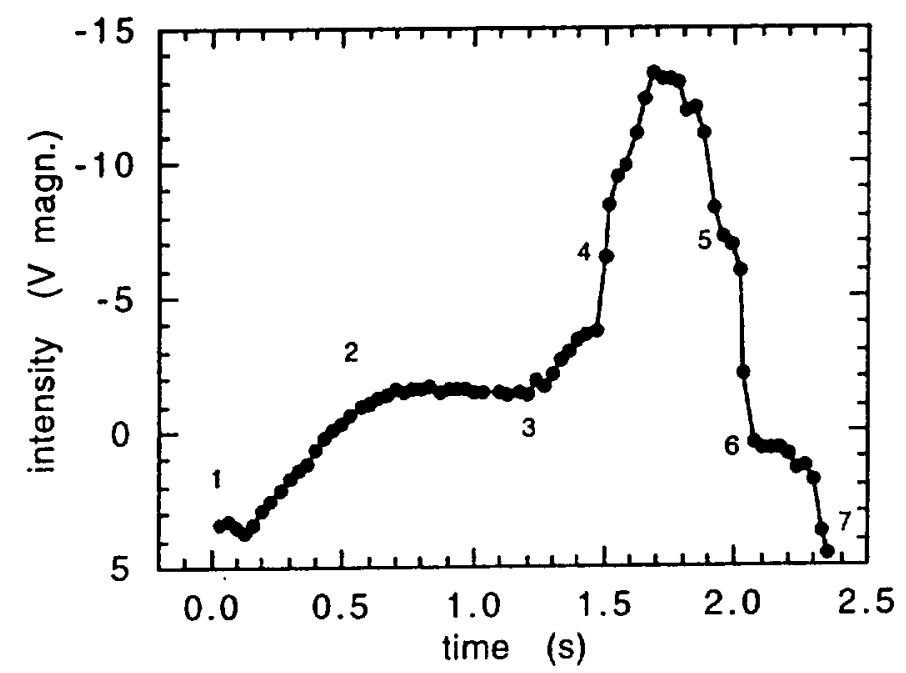

Figure 2 The visual light-curve magnitude of the meteor (normalized at $100 \mathrm{~km}$ distance) versus time. Markers as shown in Figure 1.

\section{The observations}

\subsection{THE FIREBALL}

The fireball was observed by a wide angle Mullard XX1332-intensified Hi-8 camera (Jenniskens, 1999) onboard ARIA (Figure 1). The video record was digitized with $640 \times 480$ resolution elements. Figure 1 is taken from a single frame shortly after the bright flare. The full frame is shown in Borovicka and Jenniskens (2000). Note that a small fragment of the meteor survives to the low altitude marker [7]. The field of view of the image is about $20 \times 25$ degrees. The meteor entered the frame at the top of the field as a faint point source (marker [1]). It soon spreads into the characteristic V-shaped structure first recognized by Spurny $e t$ al. (2000a). Shortly after, it turns into a droplet shape and at the same 


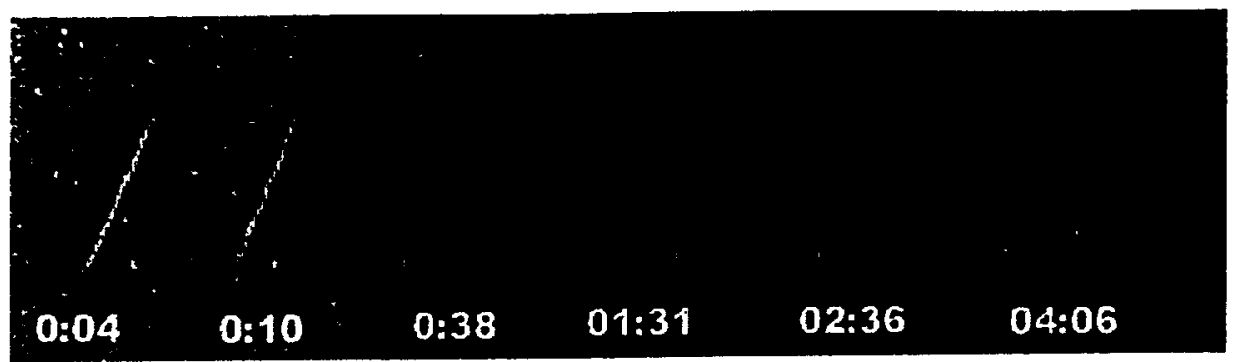

Figure 3 The persistent train as seen from ARIA at different times following the meteor (in minutes and seconds).

After about 1.5 minutes from the time of the meteor (1:31 in Figure 3) the basic shape of a " 2 " is formed as a composite of the middle curl and the foot of the top curl. Because of its striking shape and occurrence, this particular nature's-own end-of-the-millennium fireworks was soon named the "Y2K train". The "2"-shaped feature, including its many 'billowing' features, does not significantly change over the next 12 minutes (Figure 5). Aircraft motion causes a gradual, but not substantial, drift in azimuth as seen by the projection of the train against the star background (Figure 3). The train was observed until 04:13:29 UT, when it drifted outside the field of view of the ARIA camera.

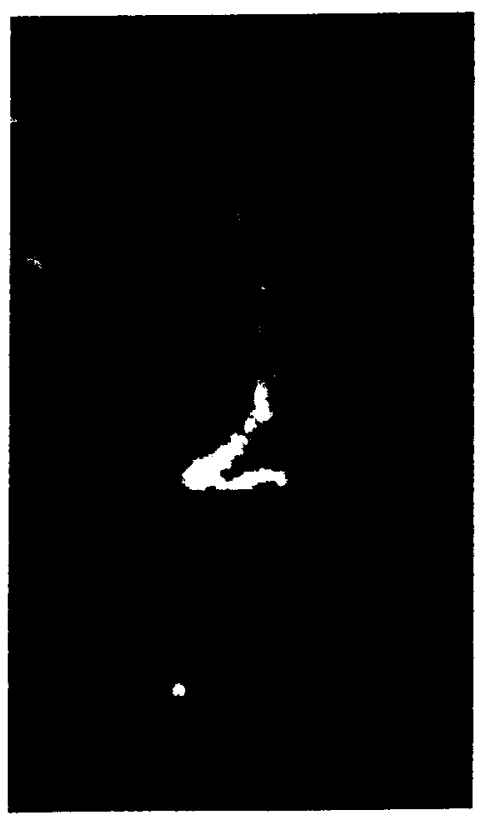

Figure 4. First view from FISTA 50 seconds after fireball (camera: FH5OR). Notice the corkscrew pattern.

In response to the unusual sighting, the FISTA aircraft quickly changed its direction in order to bring this train into view of its onboard cameras and spectrometers. The earliest record is taken with the upward looking camera FH50R, only 48 seconds after the meteor appeared (Figure 4). The corkscrew pattern is clearly visible with most of the light intensity now being in the middle and upper parts of the train. 
The lowest visible part of the train (at time 04:06) is at about $79 \mathrm{~km}$ altitude.

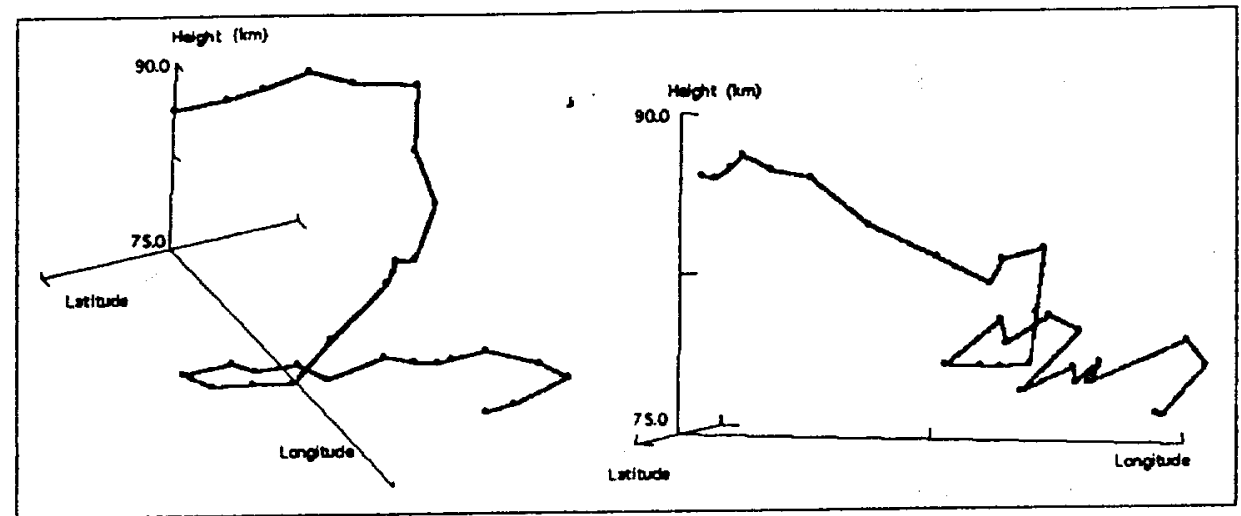

Figure 6. The 3-dimensional structure of the train at 04:04:35 UT, at time 04:06 after the meteor from two perspectives. The positions of train features are indicated.

TABLE I

\begin{tabular}{llllllll}
\hline$\#$ & $\begin{array}{l}\mathrm{H} \\
(\mathrm{km})\end{array}$ & $\begin{array}{l}\mathrm{R}(\mathrm{ARIA}) \\
(\mathrm{km})\end{array}$ & $\begin{array}{l}\mathrm{R}(\mathrm{FISTA}) \\
(\mathrm{km})\end{array}$ & $\begin{array}{l}\# \\
(\mathrm{~km})\end{array}$ & $\mathrm{H}$ & $\begin{array}{l}\mathrm{R}(\text { ARIA }) \\
(\mathrm{km})\end{array}$ & $\begin{array}{l}\mathrm{R}(\text { FISTA) } \\
(\mathrm{km})\end{array}$ \\
\hline 1 & $91.6 \pm 3$ & $308 \pm 6$ & $191 \pm 4$ & 16 & 82.6 & 320 & 203 \\
2 & 90.9 & 304 & 189 & 17 & 84.2 & 320 & 205 \\
3 & 91.0 & 303 & 188 & 18 & 83.2 & 319 & 204 \\
4 & 91.0 & 300 & 187 & 19 & 83.8 & 318 & 205 \\
5 & 90.2 & 302 & 191 & 20 & 83.2 & 318 & 205 \\
6 & 89.7 & 296 & 186 & 21 & 80.3 & 309 & 197 \\
7 & 88.4 & 300 & 189 & 22 & 81.3 & 310 & 199 \\
8 & 86.7 & 302 & 191 & 23 & 80.5 & 309 & 198 \\
9 & 85.5 & 312 & 195 & 24 & 81.4 & 309 & 199 \\
10 & 86.3 & 309 & 197 & 25 & 80.5 & 306 & 196 \\
11 & 86.6 & 312 & 201 & 26 & 81.7 & 308 & 200 \\
12 & 85.9 & 316 & 202 & 27 & 80.5 & 307 & 200 \\
13 & 82.1 & 318 & 203 & 28 & 79.2 & 308 & 199 \\
14 & 82.1 & 318 & 203 & 29 & 79.3 & 310 & 200 \\
15 & 82.4 & 331 & 216 & & & & \\
\hline
\end{tabular}


the train gradually decline in elevation, which is mostly an effect of the aircraft moving away from the train. The expected effect is an increase in zenith distance by about $0.012 \mathrm{deg} / \mathrm{s}$. The upper part, above marker point 14 (above the foot of the "2") stays at nearly constant elevation. This implies that the train drifts either towards FISTA (in west/southwestward direction) at a rate of about $70 \mathrm{~m} / \mathrm{s}$, or this part of the train moves upward at a rate of about $25 \mathrm{~m} / \mathrm{s}$, relative to the train below marker 14 . A relative $75 \mathrm{~m} / \mathrm{s}$ west/northwestward drift is implied by the shape of the train in Figure 6, suggesting that most of the effect is in fact due to a horizontal wind gradient with altitude.

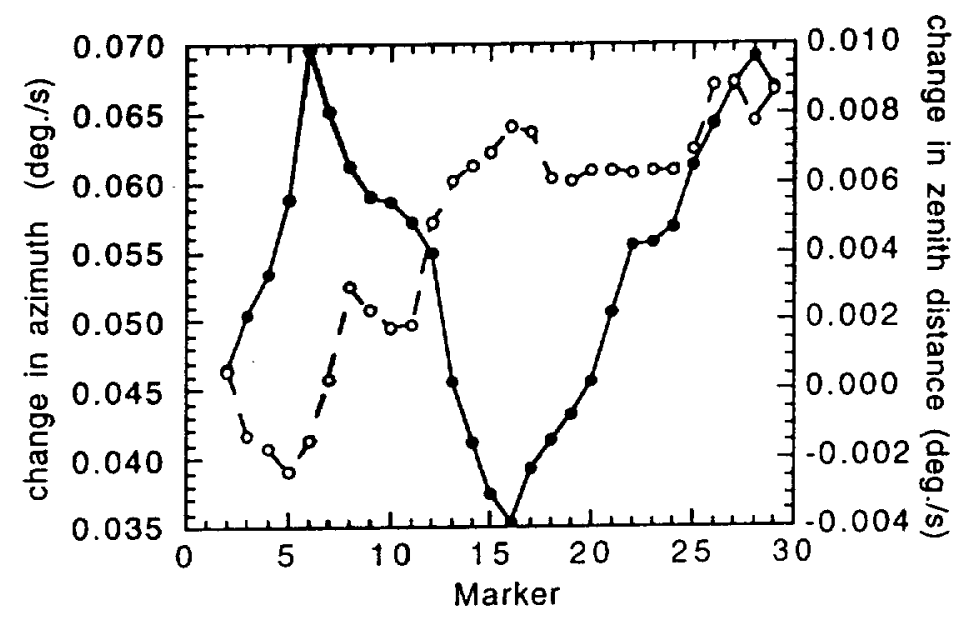

Figure 8. Mean rate of change in azimuth and zenith distance between times $03: 38$ and 08:20.

\subsection{TRIANGULATION OF THE METEOR}

By extrapolating the train motion backward in time, we can reconstruct the position of the meteor as seen from FISTA and calculate the trajectory by comparison with the meteor record from ARIA (Figure 1). We use the fact that the FISTA aircraft motion was almost linear during the period of interest (03:38-08:20), as shown in Figure 9. Figure 10 shows the resulting position of each marker point, in azimuth and zenith distance as seen from FISTA. We show the results for both linear and a parabolic extrapolations of the observed trend of position versus time for each feature in the train. 
additional constraint, because it defines the length of the observed trajectory and thus the position of the meteor's path in distance from the aircraft. No reasonable solution is obtained for a fit differing more than \pm 0.4 degree in zenith distance from that shown as a dashed line in Figure 10. If the zenith distance is as low as implied by the data in Figure 10 the computed solution for the speed of the meteor will be too high and hence the trajectory too far from both aircraft.

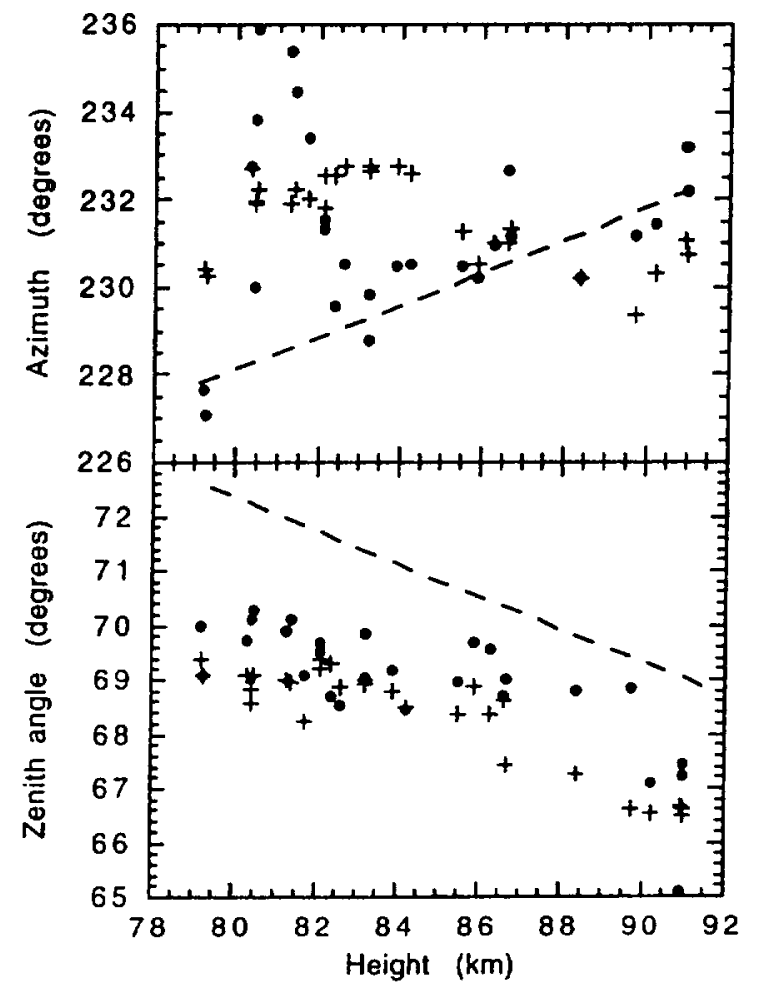

Figure 10. Azimuth and zenith distance angle of the meteor itself as seen from FISTA. The position of the meteor is derived from assumed linear (+) or parabolic $(\bullet)$ extrapolation of train drift. Dashed lines show the trajectory that provides the best triangulation results with ARIA.

The reason for this discrepancy remains unknown. However, the best fitted meteor trajectory (dashed line) is in good agreement with other Leonid fireball trajectories, with key features at similar altitudes. The meteor discussed here was first detected at about $195 \mathrm{~km}$ altitude and ended the so-called 'diffuse phase' (Spurny et al., 2000a) at about 136 


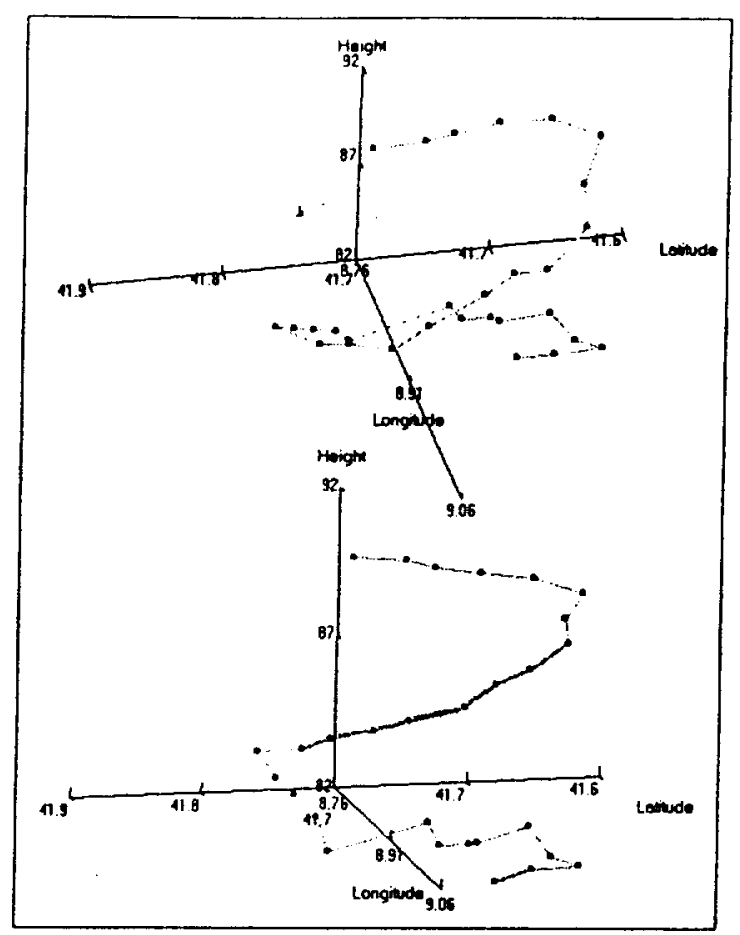

Figure 11. Model of the Y2K train at 04:04:35 UT by imposing a periodic wind variation with altitude but without a wind gradient.

Radar wind measurements in middle-Europe (Singer et al., 2000) showed tidal wind oscillations with an amplitude of about $40 \mathrm{~m} / \mathrm{s}$ and a scale height of about $8.5 \mathrm{~km}$ between 85 and $105 \mathrm{~km}$ altitude. At $04 \mathrm{UT}$, the zonal winds were changing in direction from east to west, more quickly at higher altitude. These observations are consistent with the observed east-west gradient in Figure 6.

\section{Acknowledgements}

We are grateful for the constructive reviews by Jack Drummond and Frans Rietmeijer to improve the presentation of this paper. Klaas Jobse operated the Mullard XX1332-intensified Hi-8 camera onboard ARIA. Mike Koop operated the FH50R camera on FISTA. The 1999 Leonid MAC was sponsored by the NASA Exobiology, Planetary Astronomy, 